\title{
Artificial restriction DNA cutter for site-selective gene insertion in human cells
}

\author{
Kenichiro Ito, ${ }^{\mathrm{a}}$ Narumi Shigi ${ }^{\mathrm{a}, \mathrm{b}}$ and Makoto Komiyama ${ }^{\mathrm{a}, \mathrm{b}, *}$ \\ Received (in $X X X, X X X) X_{\text {th }} X X X X X X X X X 20 X X$, Accepted $X$ th $X X X X X X X X X 20 X X$ \\ ${ }_{5}$ DOI: 10.1039/b000000x
}

With the use of chemistry-based artificial restriction DNA cutter (combination of Ce(IV)/EDTA and a pair of pcPNA), both an antibiotic-resistance gene and a fluorescent reporter protein gene were incorporated into the targeted site through ${ }_{10}$ homologous recombination in human cells.

Homologous recombination is one of the most powerful methods to manipulate genomes, and has been attracting significant interests of chemists, biochemists, and biologists. The site of recombination can be dictated by DNA cutter, since a double 15 strand break (DSB) at target site notably activates the DNA repair machinery there. ${ }^{1}$ Various kinds of protein-based DNA cutters have been reported to promote homologous recombination. Typical examples are $\mathrm{ZFN}^{2-5}$ and TALEN, ${ }^{6-8}$ in which a nonspecific nuclease domain of FokI restriction enzyme was fused 20 with either tandemly-assembled zinc finger proteins or a transcription activator-like effector. Homing endonucleases which recognize long sequences were engineered. ${ }^{9-12}$ Recently, a CRISPR/CAS method based on RNA-guided DNA scission was also developed as a new gene manipulation method. ${ }^{13-15}$ However,

${ }_{25}$ few reports have been made on completely chemistry-based tools for homologous recombination.

Recently, we prepared artificial restriction DNA cutter (ARCUT), which is composed of Ce(IV)/EDTA complex and a pair of pseudo-complementary peptide nucleic acid (pcPNA) 30 strands and never involves either proteins or other biomolecules. ${ }^{16,17}$ PNA is a synthetic DNA analog which is formed from poly[ $N$-(2-aminoethyl)glycine] backbone. The site of selective scission of this cutter is a priori determined by Watson-Crick base-pairings between the pcPNA strands and the ${ }_{35}$ DNA substrate. Moreover, this cutter can be rapidly and easily prepared by well-established chemical method. It was also shown that DSB introduced by ARCUT is satisfactorily recognized by the repair system in human cells and stimulates the homologous recombination. ${ }^{18,19}$ For example, the gene of blue fluorescent 40 protein (BFP) was converted in the cells to that of highly relevant enhanced green fluorescent protein (EGFP). This mutation involved the replacement of the sequence at the chromophoreforming site of BFP with another sequence of the same length which is presented by the EGFP gene in the donor DNA. In this 45 paper, ARCUT-mediated homologous recombination is used to insert various genes to predetermined sites in human cells. The efficiency of insertion is sufficiently high, although the DNA donors employed here are far longer than those for the mutation experiments. The inserted neomycin-resistance gene $\left(\mathrm{Neo}^{\mathrm{r}}\right)$ and ${ }_{50}$ DsRed2 gene are successfully expressed to show the expected functions (the antibiotic resistance or fluorescence emission). Versatile utility of ARCUT for gene manipulation has been further indicated.

a)

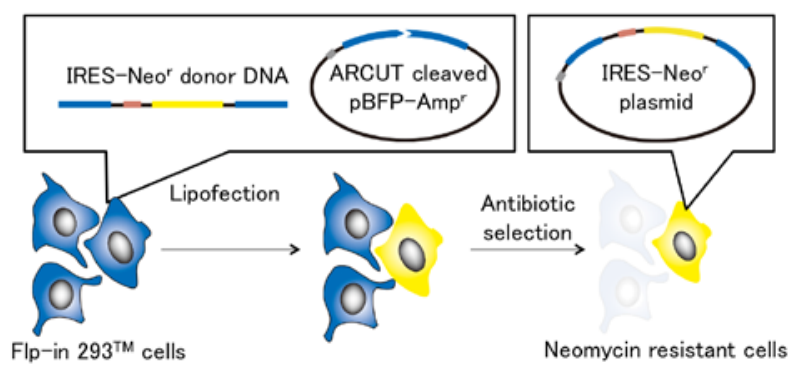

b)

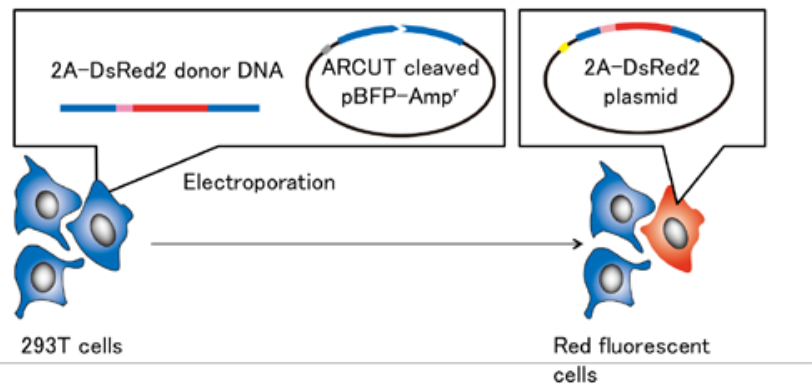

55 Fig. 1 Schemes for the insertion of target gene into a plasmid in human cells through homologous recombination. (a) Insertion of neomycinresistance gene $\left(\mathrm{Neo}^{\mathrm{r}}\right)$. The BFP gene in $\mathrm{pBFP}-\mathrm{Amp}^{\mathrm{r}}$ was cut by ARCUT and incorporated into human cells, together with a donor involving IRES$\mathrm{Neo}^{\mathrm{r}}$ sequence. The cells growing in the presence of G418 are selected 60 and analyzed. (b) Insertion of DsRed2 gene. The donor involves 2ADsRed2 sequence, and thus the cells emitting red fluorescence are counted.

Outline of the present study is shown in Fig. 1. A BFP gene is ${ }_{65}$ placed in a plasmid $\mathrm{pBFP}-\mathrm{Amp}^{\mathrm{r}}$, which has no neomycinresistance gene. First, the BFP gene is cut by ARCUT at the chromophore-forming site. Then, this DNA product is introduced into human cells, together with a donor involving either IRES$\mathrm{Neo}^{\mathrm{r}}$ (Fig. 1a) or 2A-DsRed2 sequence (Fig.1b). In these donors, 70 either $\mathrm{Neo}^{\mathrm{r}}$ or DsRed2 gene has no promoter and thus cannot be directly expressed. The homology region in the donor covers 
about 190 bp of the upstream of the ARCUT scission site and 510-540 bp of its downstream. When the corresponding homologous recombination occurs in the human cells, IRES-Neo ${ }^{\mathrm{r}}$ gene cassette (1608 bp) or 2A-DsRed2 gene cassette (754 bp) is 5 inserted to the plasmid. In the upstream of $\mathrm{Neo}^{\mathrm{r}}$, IRES (internal ribosome entry site; $495 \mathrm{bp}$ ) is placed to recruit the ribosome in the cells and allow the $\mathrm{Neo}^{\mathrm{r}}$ to be translated independently from the typical cap-dependent translation pathway (Fig. 1a). ${ }^{20}$ On the other hand, 2A peptide sequence is placed in the upstream of the 10 DsRed2 gene (Fig. 1b). This short peptide (21 amino acids) shows self-cleaving activity during ribosomal protein synthesis, and can be an attractive alternative to IRES. ${ }^{21}$ The incorporation and expression of the $\mathrm{Neo}^{\mathrm{r}}$ gene can be detected in terms of the resistance of the human cells to G418, whereas the DsRed2 gene 15 in the cells should emit the red fluorescence. Further details on the constructs are shown in Fig. S1.

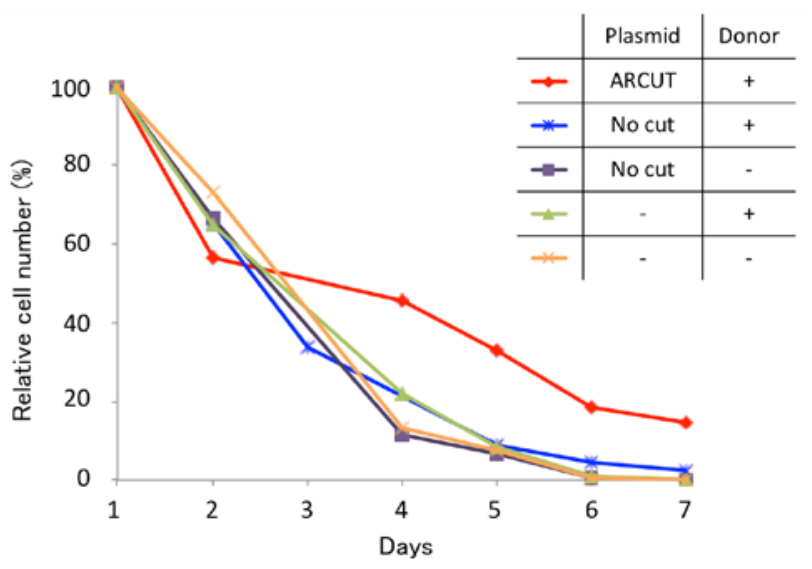

Fig. 2 Survival rates of variously treated human cells in the medium containing G418. The red line corresponds to the experiment in which 20 PBFP-Amp ${ }^{\mathrm{r}}$ plasmid was cut by the ARCUT and incorporated into the cells together with the donor involving the IRES-Neo ${ }^{r}$ sequence (see Fig. 1a). As references, pBFP-Amp ${ }^{r}$ which was not treated by the ARCUT was lipofected into the cells with (blue) or without the donor (purple). The green line is for the experiment where only the donor was transfected to 25 the cells in the absence of pBFP-Amp ${ }^{\mathrm{r}}$. These cells were cultured in the presence of $1.2 \mathrm{mg} / \mathrm{ml}$ of G418.

With the use of the ARCUT presented in Fig. S2, the pBFP$\mathrm{Amp}^{\mathrm{r}}$ plasmid was selectively cut at the chromophore-forming 30 site in the BFP gene. Then, the product of this ARCUT scission was incorporated into Flp-in $293^{\mathrm{TM}}$ cells (Life Technologies) by lipofection using FuGENE HD (Promega), together with the donor DNA involving the IRES-Neo ${ }^{r}$ sequence (Fig. 1a). The cells were cultured in the medium containing G418. This 35 antibiotic inhibits protein elongation event of ribosome in eukaryote and is used as a selective antibiotic for neomycinresistance gene encoding aminoglycoside 3'-phosphotransferase. As shown by the red line in Fig. 2, thus treated cells are much more resistant to G418, and even after 7 days, significant 40 proportions of the cells are still alive. With the lipofection of (i) the plasmid only, (ii) the donor only, or (iii) the diluent only, however, all the cells died under the same conditions. By introducing the ARCUT scission product and the IRES-Neo ${ }^{r}$ donor, the targeted homologous recombination successfully ${ }_{45}$ occurred in the human cells to insert the $\mathrm{NeO}^{\mathrm{r}}$ gene to the DSB site. Consistently, the survival rate of the cells was much lower when pBFP-Amp ${ }^{r}$ was lipofected into the cells without being cut by the ARCUT, together with the donor DNA (the blue line). Apparently, the ARCUT-induced DSB notably promotes the so targeted insertion of the IRES-Neo ${ }^{\mathrm{r}}$ gene cassette (1608 bp) through homologous recombination. The gradual decrease in the survival rate even with the introduction of ARCUT product/IRES-Neo ${ }^{\mathrm{r}}$ donor is due to the fact that the Flp-in $293^{\mathrm{TM}}$ cells have no plasmid-duplicating activity and thus the fraction of 55 the cells containing the recombinant plasmid(s) decreases as the cell division proceeds.

The efficiency of gene insertion by ARCUT was more directly measured by inserting DsRed2 gene as fluorometric probe (Fig. 1b). Into $293 \mathrm{~T}$ cells, the ARCUT-scission product and 60 the donor involving the 2A-DsRed2 sequence were electroporated. The electroporation was achieved on a Neon ${ }^{\mathrm{TM}}$ Transfection System from Life Technologies (Square wave, Pulse Voltage $1150 \mathrm{~V}$, Pulse Width $20 \mathrm{~ms}$, and Pulse Number 2). After 36 hours, the cells were analyzed by flow cytometry (Guava ${ }_{65}$ EasyCyte Plus, Millipore). As shown in Fig. 3a (and also in Fig. S3a), many cells emit red fluorescence, only when the pBFP$\mathrm{Amp}^{\mathrm{r}}$ was first cut with the ARCUT and introduced to the cells together with the 2A-DsRed2 donor (the left bar). When the intact $\mathrm{pBFP}-\mathrm{Amp}^{\mathrm{r}}$ and the donor are introduced to the cells (No 70 cut), the fluorescent cells are marginal (the middle bar). Upon the introduction of the donor DNA alone (without the pBFP-Amp ${ }^{r}$ ), few fluorescent cells were observed as expected (the right bar). The importance of the site-selective scission by the ARCUT for the present insertion was further evidenced. The sequencing 75 experiments were also consistent with the designed gene insertion (data not presented).

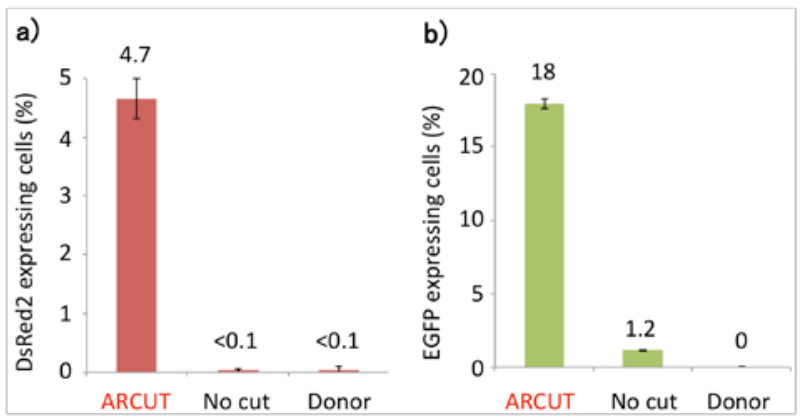

Fig. 3 (a) Evaluation of the insertion efficiency by flow cytometry for the ARCUT-mediated insertion of DsRed2 gene using the 2A-DsRed2 donor (according to Fig. 1b). In (b), the efficiency of ARCUT-mediated 80 mutation (conversion of BFP gene to EGFP gene) under comparable conditions is presented for the purpose of comparison (see the text for details). In both (a) and (b), the bars "Not cut" are for the experiments where the plasmid was not treated with ARCUT and transfected into the cells together with the corresponding donor. In the bars "Donor", only the 85 donor (without the plasmid) was transfected into the cells.

It has now been concluded that both the insertion of the whole of a gene and the mutation (replacement of rather short sequence in a gene) can be promoted by ARCUT-mediated homologous 90 recombination. Thus, we decided to compare the efficiencies of these two reactions under comparable conditions. For the mutation experiment, the conversion of BFP to EGFP was chosen. ${ }^{18,19}$ The same amounts of the ARCUT-scission product of 
pBFP-Amp ${ }^{\mathrm{r}}$ and the donor DNA were used (4.8 nM and $34 \mathrm{nM}$, respectively), and the lengths of the homology region were also made similar (compare b and c in Fig. S1). As shown in Fig. 3b, the mutation (the conversion of BFP to EGFP) occurred slightly 5 more efficiently than the insertion of DsRed2 in 24 hours. However, the difference is rather small (only 4 fold here), when it is considered that the 2A-DsRed2 insert is almost 750 bp long and could make unfavorable steric effects on the homologous recombination.

10 In conclusion, desired genes can be successfully inserted to targeted site through ARCUT-mediated homologous recombination. This fact indicates further potentiality of this chemistry-based DNA cutter as tools for genome manipulation.

15 The authors should like to thank Prof. Hiroshi Ueda of Tokyo Institute of Technology for kindly providing the IRES-Neo ${ }^{r}$ cassette. This work was partially supported by Grants-in-Aid for Specially Promoted Research from the Ministry of Education, Science, Sports, Culture and Technology, Japan (18001001 and 20 22000007).

\section{Notes and references}

${ }^{a}$ Research Center for Advanced Science and Technology, The University of Tokyo, 4-6-1 Komaba, Meguro-ku, Tokyo, 153-8904 Japan.

${ }^{b}$ Life Science Center of Tsukuba Advanced Research Alliance, University

25 of Tsukuba, 1-1-1 Tennoudai, Tsukuba, Ibaraki, 305-8577 Japan.

E-mail: komiyama@tara.tsukuba.ac.jp

$\dagger$ Electronic Supplementary Information (ESI) available: [Constructs used for the insertion and the mutation, structure of ARCUT as well as its selective scission, and the results of ARCUT-mediated mutation of BFP 30 to EGFP]. See DOI: 10.1039/b000000x/

1 P. Rouet, F. Smih and M. Jasin, Proc. Natl. Acad. Sci. USA., 1994, 91, 6064-6068.

2 Y. G. Kim, J. Cha and S. Chandrasegaran, Proc. Natl. Acad. Sci.

35 USA., 1996, 93, 1156-1160.

3 M. H. Porteus and D. Baltimore, Science, 2003, 300, 763.

4 F. D. Urnov, J. C. Miller, Y. L. Lee, C. Beausejour, J. M. Rock, S. Augustus, A. C. Jamieson, M. H. Porteus, P. D. Gregory and M. C. Holmes, Nature, 2005, 435, 646-651.

405 T. Mashimo, A. Takizawa, B. Voigt, K. Yoshimi, H. Hiai, T. Kuramoto and T. Serikawa, PLoS One, 2010, 5, e8870.

6 M. Christian, T. Cermak, E. L. Doyle, C. Schmidt, F. Zhang, A. Hummel, A. J. Bogdanove and D. F. Voytas, Genetics, 2010, 186, 757-761.

457 M. M. Mahfouz, L. Li, M. Shamimuzzaman, A. Wibowo, X. Fang and J. K. Zhu, Proc. Natl. Acad. Sci. USA., 2011, 108, 2623-2628.

8 C. Mussolino, R. Morbitzer, F. Lütge, N. Dannemann, T. Lahaye and T. Cathomen, Nucleic Acids Res., 2011, 39, 9283-9293.

9 B. L. Stoddard, Q. Rev. Biophys., 2005, 38, 49-95.

5010 J. C. Epinat, S. Arnould, P. Chames, P. Rochaix, D. Desfontaines, C. Puzin, A. Patin, A. Zanghellini, F. Pâques and E. Lacroix, Nucleic Acids Res., 2003, 31, 2952-2962.

11 J. B. Doyon, V. Pattanayak, C. B. Meyer, and D. R. Liu, J. Am. Chem. Soc., 2006, 128, 2477-2484.

5512 F. Paques and P. Duchateau, Curr. Gene Ther., 2007, 7, 49-66.

13 M. Jinek, K. Chylinski, I. Fonfara, M. Hauer, J. A. Doudna and E. Charpentier, Science, 2012, 337, 816-821.

14 L. S. Qi, M. H. Larson, L. A. Gilbert, J. A. Doudna, J. S. Weissman, A. P. Arkin and W. A. Lim, Cell, 2013, 152, 1173-1183.

6015 W. Y. Hwang, Y. F. Fu, D. Reyon, M. L. Maeder, S. Q. Tsai, J. D. Sander, R. T. Peterson, J. R. J. Yeh and J. K. Joung, Nat. Biotech., 2013, 31, 227-229.

16 M. Komiyama, Y. Aiba, Y. Yamamoto and J. Sumaoka, Nat. Protoc., 2008, 3, 655-662.
6517 H. Katada and M. Komiyama, Chembiochem, 2009, 10, 1279-1288.

18 H. Katada, H. Chen, N. Shigi and M. Komiyama, Chem. Commun., 2009, 6545-6547.

19 H. Katada, T. Harumoto, N. Shigi and M. Komiyama, Nucleic Acids Res., 2012, 40, e81.

7020 S. D. Baird, M. Turcotte, R. G. Korneluk and M. Holcik, RNA, 2006, 12, $1755-1785$

21 G. Trichas, J. Begbie and S. Srinivas, BMC Biol., 2008, 6, 40. 